\title{
Investigation of thermo-oxidative ageing effects on the fatigue design of automotive anti-vibration parts
}

\author{
Morgane Broudin ${ }^{1,}$, Yann Marco ${ }^{1}$, Vincent Le Saux ${ }^{1}$, Pierre Charrier ${ }^{2}$, Wilfried Hervouet $^{2}$ and Pierre-Yves Le Gac ${ }^{3}$ \\ ${ }^{1}$ ENSTA Bretagne, Institut de Recherche Dupuy de Lôme (IRDL), UMR CNRS 6027, 2 Rue François Verny, 29200, Brest, France \\ ${ }^{2}$ Vibracoustic, CAE \& Durability Prediction, 1 Rue du Tertre, 44474, Carquefou, France \\ ${ }^{3}$ IFREMER (French Ocean Research Institute), Centre de Brest, Materials and Structures Group, BP 70, F-29280, Plouzané, France
}

\begin{abstract}
Elastomeric parts found in automotive anti-vibration systems are usually massive. Ageing therefore leads to heterogeneous properties, usually induced by several mechanisms due to the availability, or not, of oxygen in the part's bulk. To better understand the effects of oxygen in the degradation process and on the fatigue properties, this paper aims at studying the ageing of a rubber material (semi-efficient vulcanization system) in aerobic (with oxygen) and anaerobic (without oxygen) conditions for a wide range of temperatures, relevant for under hood applications. The material studied here is a fully formulated compound NR/IR blend reinforced with carbon black. A specific protocol to perform ageing under anaerobic conditions was set up and validated. Numerous tests have been carried out to evaluate the consequences of ageing on monotonic tension and fatigue properties. A comparison of these consequences and of their kinetics is finally presented for ageing with or without oxygen.
\end{abstract}

\section{Introduction}

\subsection{Industrial context}

Rubber parts for anti-vibration systems applications need to be designed taking into account several parameters: efficient damping on given ranges of frequencies, reliable static response over the service time, resistance to creep and fatigue [1-2]. The automotive applications require, in addition, the assessment of these features for thermal conditions inducing ageing. This ageing is related both to the effect of oxygen but also, for these massive parts, to the effect of temperature only. Several recent features illustrate the need to go beyond the empirical rules. The first one is an ever reducing development time, which leads to assess compounds faster than ever. A second point is the need to design parts for worldwide purposes increasing the severity profiles of users (roads and weather conditions). A last one is connected to the environmental standards (REACH and EURO6) that restrict the use of specific ingredients and lead to higher service temperatures. Integrating efficiently the ageing effects in the design loop therefore becomes a crucial need.

\subsection{Scientific background}

Thermo-oxidative degradation of rubber components has been widely investigated in the literature [3-10]. Ageing could indeed induce severe modifications of the mechanical properties both in terms of constitutive response [11], failure properties under monotonic tension
[12], crack propagation [13] and duration life [14]. Nevertheless, relating the degradation mechanisms to the evolution of the mechanical properties is not an easy task because there are so many mechanisms at stake. A first range of mechanisms is related to oxidation, leading to important modifications of the elastomer network (mainchain scissions, crosslink formation and/or crosslink breakage) [15]. Large-sized automotive parts age heterogeneously due to the availability, or not, of oxygen in the part's bulk. A classic analysis could be done using a DLO approach based on the competition between the kinetics of oxygen diffusion and consumption [16]. However hardness profiles along the thickness of thick parts or specimens also illustrate that ageing leads to mechanical changes even without oxygen [17]. Numerous studies investigated the effect of temperature ageing in the absence of oxygen on the mechanical properties of rubber materials. A first range of studies investigated the evolution of the tensile properties for anaerobic conditions and have found a limited increase of stiffness and little reduction of the ultimate tensile properties [1819]. Then, a change of fatigue crack growth resistance have been noticed for fully formulated compound and different vulcanization systems under various ageing environments. A higher fatigue crack growth rate is observed under air than under nitrogen whatever the vulcanization system. The oxidative degradation of the rubber causes a high reversion inducing a dominant effect on the mechanical resistance [20]. This effect was confirmed later to a lesser extent [21].

There is limited data available on the effect of thermooxidative ageing on fatigue properties. Moreover the

\footnotetext{
*Corresponding author: morgane.broudin@ensta-bretagne.fr
} 
origins of the evolutions of mechanical properties both for stiffness, failure or fatigue still remain unclear. Several explanations could be suggested: plasticizers diffusionevaporation [22], sulfur network evolution [10,17], fillers network evolution [23] and/or evolution of the interface rubber/fillers [24].

The present paper aims providing an extensive comparison of the mechanical consequences of ageing under anaerobic and aerobic conditions, based on the results of monotonic tensile tests and fatigue tests. Secondly, the goal is to better understand the common mechanism at stake under anaerobic and aerobic ageing conditions and to investigate the hypothesis of a physical ageing induced by the loss of low molecular species.

\section{Experimental settings}

\subsection{Materials}

\subsubsection{Fully formulated compound}

The material studied here is a carbon black-filled NR/IR blend. All samples were made using the same material batch to limit properties scattering due to mixing. This material will be called " $C$ " in the Figures presented in the following.

\subsubsection{Simplified compound}

To determine the influence of low molecular weight components including plasticizers on the durability, it would be very convenient to consider the same compound but without these ingredients. Nevertheless, a filled material, as the material $\mathrm{C}$, could not be mixed without plasticizers. An extraction of low molecular weight components was therefore performed thanks to swelling in toluene. Then, the specimen is dried at room temperature to get back to a geometry close to the initial one. This material will be called " $\mathrm{B}$ " in the Figures hereafter.

\subsection{Accelerated ageing conditions}

\subsubsection{Thermo-oxidative ageing}

Thermo-oxidative ageing was carried out in ovens with air-flow control (Fig. 1). To get reliable tests, several authors noticed the importance to specify the ageing conditions, such as air speed and air exchanges rate [25]. Therefore, to have quite similar ageing conditions, all used ovens have been checked in terms of air speed and air renewal rates thanks to an anemometer [26]. A thermocouple checks the temperature close to the samples and is connected to continuous strip-chart recorders. The table 1 gives the tested temperatures and durations.

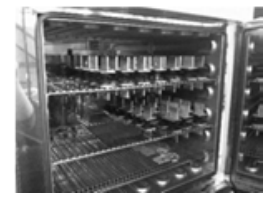

Fig. 1. Picture of the specimens in the oven during thermooxidative ageing.

Table 1. Thermo-oxidative ageing conditions.

\begin{tabular}{|c|c|c|c|c|c|c|c|}
\hline \multicolumn{8}{|c|}{ Ageing temperature $\left[{ }^{\circ} \mathrm{C}\right]$} \\
\hline & 40 & 50 & 60 & 80 & 100 & 110 & 120 \\
\hline \multirow{13}{*}{ 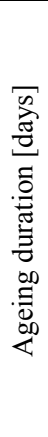 } & \multirow{13}{*}{$\begin{array}{l}21 \\
42\end{array}$} & & & & & & 0.25 \\
\hline & & & & & & 0.5 & 0.5 \\
\hline & & & & 1 & 1 & 1 & 1 \\
\hline & & & & 2 & & & \\
\hline & & & & 3 & 3 & 3 & 3 \\
\hline & & & & 7 & 7 & 7 & \\
\hline & & & 14 & 14 & 14 & & \\
\hline & & 21 & 21 & 21 & 21 & & \\
\hline & & 42 & 42 & 42 & 42 & & \\
\hline & & & 90 & & & & \\
\hline & & 161 & 161 & & & & \\
\hline & & 362 & 316 & & & & \\
\hline & & 771 & & & & & \\
\hline
\end{tabular}

\subsubsection{Thermal ageing}

An experimental protocol was set up to achieve thermal ageing. To package the specimens, a multilayer film is used (SIDEC CA40 NF H 00310 Class IV) that is impermeable to oxygen and temperature resistant. To avoid bending / torsion when the vacuum is performed, wedges are put on the samples (Fig. 2, on the left). To avoid any preload due to differential thermal expansions, the wedges material is chosen to present a thermal expansion coefficient close to the one of rubber. Finally, to remove oxygen inside the package, a vacuum chamber machine is used (Audionvac VMS 133) (Fig. 2, on the right). The Table 2 sums up the tested temperatures and durations for thermal ageing.
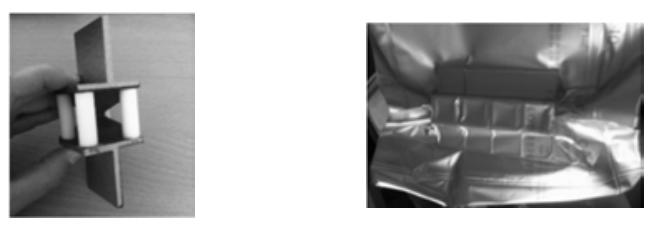

Fig. 2. AE2 specimen with PTFE wedges (left); sealed package of AE2 specimens for ageing without oxygen (right).

Table 2. Thermal ageing conditions.

\begin{tabular}{|c|c|c|c|c|c|c|c|}
\hline \multicolumn{8}{|c|}{ Ageing temperature $\left[{ }^{\circ} \mathrm{C}\right]$} \\
\hline \multirow{8}{*}{ 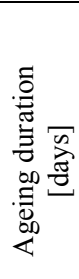 } & 40 & 50 & 60 & 80 & 100 & 110 & 120 \\
\hline & & & & & & 0.5 & 0.5 \\
\hline & & & & 3 & 3 & 3 & 3 \\
\hline & & & & 14 & 14 & & \\
\hline & & & 21 & 21 & 21 & & \\
\hline & 42 & 42 & 42 & & & & \\
\hline & & & & 90 & & & \\
\hline & & 204 & 204 & & & & \\
\hline
\end{tabular}




\subsection{Tensile tests}

S2 specimens have been used. The S2 samples are cut from plates and then put in oven to perform the various ageing conditions. The tensile tests were achieved on a testing machine equipped with a $1 \mathrm{kN}$ load cell. The tests were displacement controlled (grip speed of $200 \mathrm{~mm} / \mathrm{min}$ ). An optical extensometer was used to measure the local elongation. For each ageing condition, at least three samples were tested and the results averaged [26].

\subsection{Fatigue campaign}

Diabolo shape samples are obtained by injection moulding. The fatigue tests were displacement controlled with adapted frequencies to have a limited heat build-up. All tests were performed at a controlled temperature of $23^{\circ} \mathrm{C}$. The fatigue samples geometry and the fatigue protocol have been detailed in a former publication [28]. To keep a reasonable number of samples despite the numerous ageing conditions investigated, for each condition, 5 AE2 specimens are aged and then tested in fatigue for a given enforced displacement. In this campaign, 3 levels of displacement have been studied, $4.6 \mathrm{~mm}, 8.1 \mathrm{~mm}$ and $14.7 \mathrm{~mm}$ relating respectively to local principal strains of $80 \%, 150 \%$ and $280 \%$. These strains are evaluated by FE simulations from the geometry of the unaged sample.

\section{Results and discussion}

\subsection{Thermo-oxidative ageing}

Figures 3 to 5 illustrate the results obtained for thermooxidative ageing.

Figure 3 highlights typical results obtained for tensile tests on samples aged in presence of oxygen (here for $80^{\circ} \mathrm{C}$ and several ageing durations). These observations are valid whatever the ageing temperature. One can notice a drop of the nominal strain at break and a stiffening of the mechanical response, which is a classical result and could be related to the changes of the vulcanized network. Nevertheless, it should be noticed that the evolutions in stiffness and strain at break observed for short durations is already significant. Figure 4 shows the results of the fatigue campaigns for the same ageing conditions. The drop of the duration life with the ageing durations is clearly visible. It is worth noticing that the curves keep the same slope, except for the last ageing condition, considered here to be extremely severe.

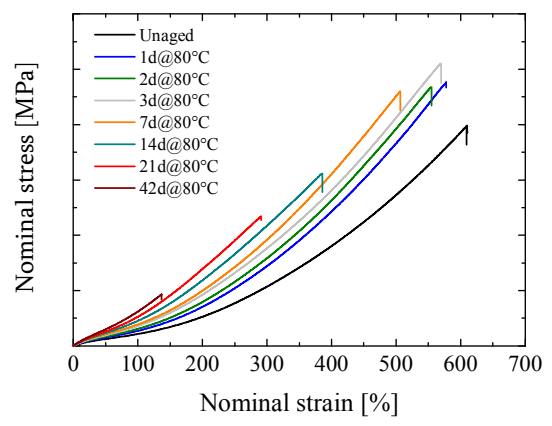

Fig. 3. Material C. Aerobic ageing. Strain - stress curves after various preliminary thermal ageing (from 1 to 42 days at $\left.80^{\circ} \mathrm{C}\right)$.

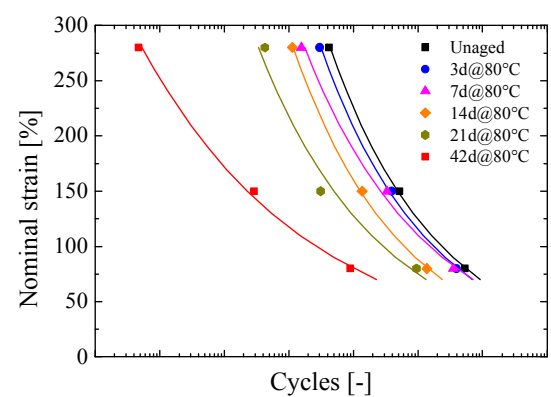

Fig. 4. Material C. Aerobic ageing. Wöhler curves after various preliminary thermal ageing (from 3 to 42 days at $80^{\circ} \mathrm{C}$ ).

A single ageing mechanism is clearly not probable for this complex ageing. Nevertheless, the identification of a time-temperature superposition seems possible, as illustrated by the master curve obtained on the relative evolution of the nominal strain at break (Fig. 5). The deduced activation energy is of $90 \mathrm{~kJ} / \mathrm{mol}$. A comparable result can be obtained from the fatigue tests with an activation energy of $95 \mathrm{~kJ} / \mathrm{mol}$ [4]. Both values are very close to other values found in the bibliography for various ageing indicators [16], [17], [29], [30].

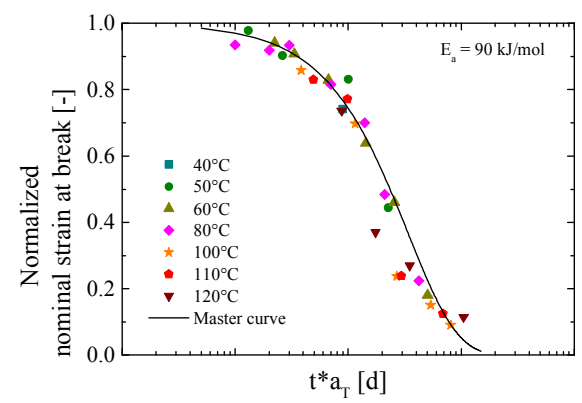

Fig. 5. Material C. Aerobic ageing. Master curve obtained on the relative evolution of the nominal strain at break. Reference temperature $=80^{\circ} \mathrm{C}$. 


\subsection{Thermal ageing}

Figures 6 to 8 illustrate the results obtained for thermal ageing conditions.

Figure 6 highlights a noticeable increase of the mechanical response for short ageing durations. This increase appears more gradually with increasing aging time. A progressive drop of failure properties is also observed. Both variations seems to occur early and then to stabilize. The stabilized values reached are less severe than for oxidative ageing. These evolutions are observed whatever the ageing temperature.

Figure 7 presents the results of the fatigue campaigns for the same ageing conditions. Here again, the drop of the duration life with the ageing duration occurs early and then stabilizes to a level much less severe than for long oxidative conditions.

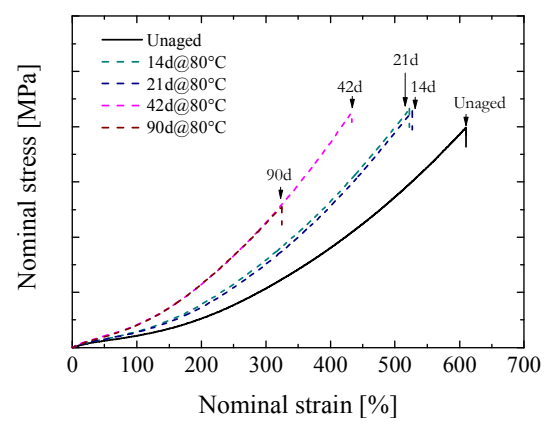

Fig. 6. Material C. Anaerobic ageing. Strain - stress curves after various preliminary thermal ageing (from 14 to 90 days at $\left.80^{\circ} \mathrm{C}\right)$.

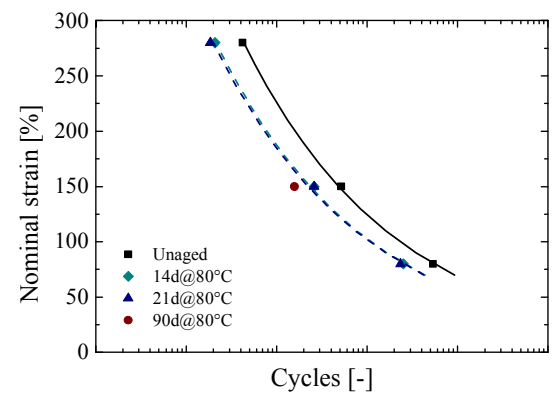

Fig. 7. Material C. Anaerobic ageing. Wöhler curves after various preliminary thermal ageing (from 3 to 90 days at $\left.80^{\circ} \mathrm{C}\right)$.

Figure 8 illustrates the master curve obtained on the relative evolution of nominal strain at break. The deduced activation energy would be of $72 \mathrm{~kJ} / \mathrm{mol}$. This value seems lower than the one obtained for thermo-oxidative aging but quite consistent with those found for anaerobic aging in the literature [31]. However, contrary to thermooxidative ageing, an identification of a time-temperature superposition from fatigue tests is not reasonable in this case.

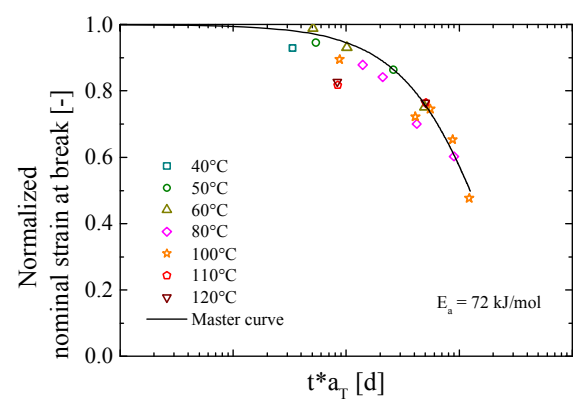

Fig. 8. Material C. Anaerobic ageing. Master curve obtained on the relative evolution of the nominal strain at break. Reference temperature $=80^{\circ} \mathrm{C}$.

\subsection{Thermal ageing vs thermo-oxidative ageing}

Figure 9 summarizes the evolution of the relative nominal strain at break for tensile tests performed on samples after aerobic and anaerobic ageing with several durations at $80^{\circ} \mathrm{C}$. Figure 10 provides the same synthesis for the variations of the relative fatigue lifetime (for a given enforced displacement).

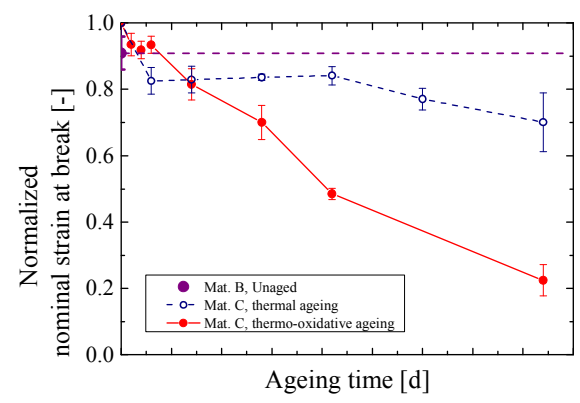

Fig. 9. Evolution of the relative nominal strain at break obtained after anaerobic and aerobic ageing at $80^{\circ} \mathrm{C}$ and after swelling in toluene (mat. B).

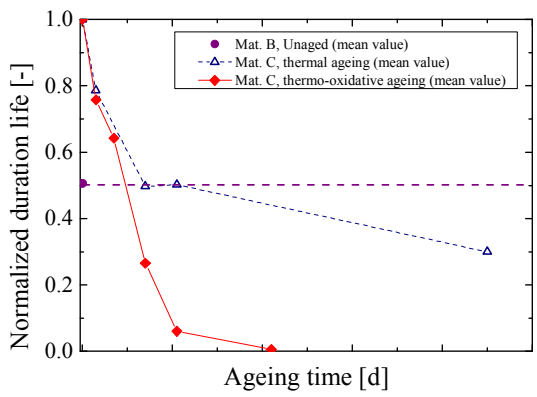

Fig. 10. Evolution of the relative nominal duration life obtained after anaerobic and aerobic ageing at $80^{\circ} \mathrm{C}$ and after swelling in toluene (mat. B). Displacement level $=8.1 \mathrm{~mm}$. 
In order to check these first comparisons, the values obtained for the samples tested after swelling in toluene (material B) were compared with the evolutions of anaerobic and aerobic ageing conditions. These comparisons are illustrated in Figures 9 and 10. The dotted line is the value obtained for the samples tested after swelling in toluene (material B).

One can observe on these curves that the drops of tensile and fatigue properties seem to present a common kinetic for the short ageing durations. Then, the loss of the failure properties reaches a stabilized level for the ageing conditions without oxygen, whereas the loss of failure properties continues for the ageing conditions with oxygen.

It should be underlined here that the results obtained are presented for $80^{\circ} \mathrm{C}$ only due to the restricted format of the paper, but similar conclusions can be done from the results obtained for lower and higher ageing temperatures as $60^{\circ} \mathrm{C}$ and $100^{\circ} \mathrm{C}$.

Figures 9 and 10 illustrate that the drop of strain at break and fatigue lifetime for anaerobic conditions stabilize for values correlated to the ones obtained for the material after swelling in toluene. It therefore seems that the initial drops of strain at break and fatigue properties observed both under aerobic and anaerobic conditions seem well correlated to the loss of low molecular weight components. The nature of these components is still to be determined even if it seems very probable that they are plasticizers. TGA and GC-MS measurements are currently done in order to clarify this point.

This evaluation performed on nominal strain at break and a given displacement for fatigue test are also valid for the global tension curve (see Figure 11) and the full fatigue curve (see Figure 12).

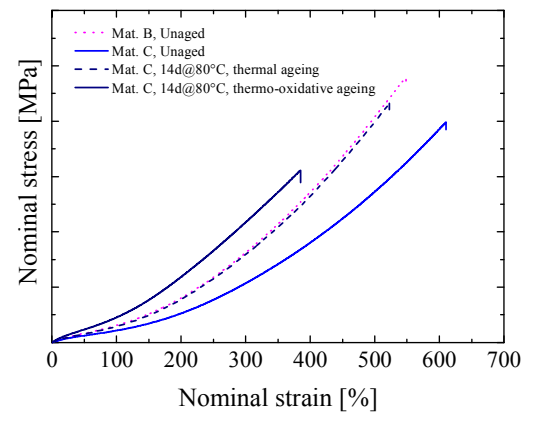

Fig. 11. Comparison of nominal stress - strain curves obtained for material $\mathrm{C}$ after anaerobic and aerobic ageing for 14 days at $80^{\circ} \mathrm{C}$ and for material $\mathrm{B}$

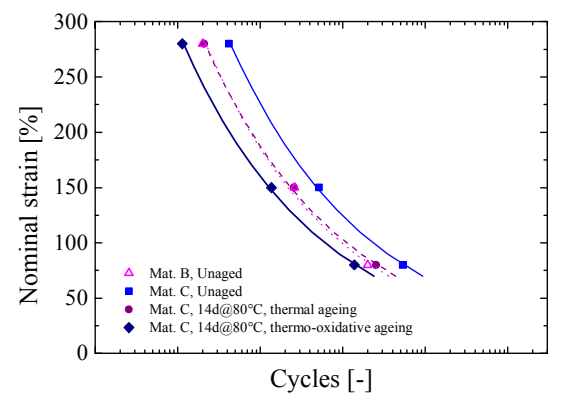

Fig. 12. Comparison of Wöhler curves obtained for material C after anaerobic and aerobic ageing for 14 days at $80^{\circ} \mathrm{C}$ and for material B.

Figure 13 compares the master curves built from the relative evolutions of the nominal strain at break for anaerobic and aerobic ageing conditions. It allows us to underline that the kinetics of aerobic ageing is substantially faster than anaerobic ageing (about a decade of difference between the two kinetics).

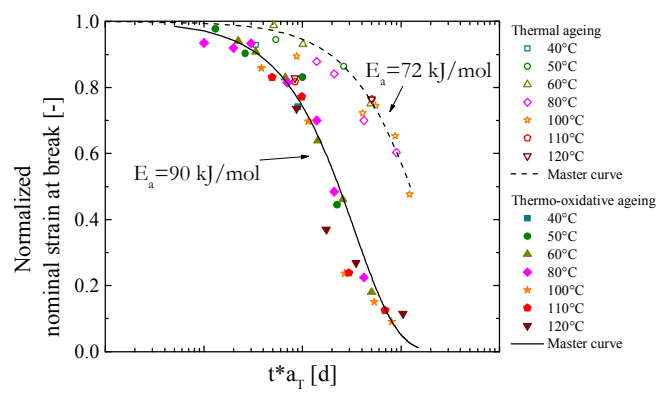

Fig. 13. Comparison of time-temperature superposition for thermal and thermos-oxidative conditions for normalized strain at break. Reference temperature $=80^{\circ} \mathrm{C}$.

\section{Conclusion and perspectives}

In this paper, we focused on the ageing of a rubber material in aerobic (with oxygen) and anaerobic (without oxygen) conditions, for a wide range of temperatures relevant for under the hood applications. Tensile and fatigue tests are analysed. For both ageing cases, progressive drops of the strain at break and the fatigue properties are observed as well as a progressive increase of the stiffness. Under anaerobic and aerobic conditions, these evolutions seem to exhibit the same kinetics for the short durations. Nevertheless, for anaerobic ageing, while ageing duration increases, the evolution of the mechanical indicators stabilizes, whereas the evolutions go on for aerobic ageing.

In order to investigate if these evolutions can be related to a physical ageing induced by the extraction of low molecular weight components (plasticizers, for example), 
tensile and fatigue tests have been performed on samples after swelling in toluene (material B). It appears that the values obtained on these samples are well correlated to the stabilization observed for anaerobic ageing. This hypothesis seems therefore reasonable and this mechanism could be common to ageing conditions with or without oxygen.

Further investigations are in progress to identify the nature of the components extracted in toluene and to understand better the other possible ageing mechanisms at stake, both for anaerobic and aerobic ageing.

\section{References}

[1] NF EN ISO 5349-1, Vibrations mécaniques Mesurage et évaluation de l'exposition des individus aux vibrations transmises par la mainPartie 1 : exigences générales (2002)

[2] NF EN ISO 5349-2, Vibrations mécaniques Mesurage et évaluation de l'exposition des individus aux vibrations transmises par la main Partie 1: guide pratique pour le mesurage sur le lieu de travail (2001)

[3] J. L. Bolland and G. Gee, Trans. Faraday Soc., 42, 236 - 243 (1946)

[4] M. Broudin, V. Le Saux, Y. Marco, P. Charrier, and W. Hervouet, Proc. Ninth Eur. Conf. Const. Models Rubber, 53 (2015)

[5] A. Herzig, M. Johlitz, and A. Lion, Proc. Ninth Eur. Conf. Const. Models Rubber, 23 (2015)

[6] S. Kamarudding, P. Y. Le Gac, Y. Marco, and A. Muhr, Proc. Seventh Eur. Conf. Const. Models Rubber, 197 (2011)

[7] C. Naumann and J. Ihlemann, Proc. Eigth Eur. Conf. Const. Models Rubber, 43 (2013)

[8] C. Schlomka, J. Ihlemann, and C. Naumann, Proc. Tenth Eur. Conf. Const. Models Rubber, 77 (2017)

[9] C. Neuhaus, A. Lion, M. Johlitz, P. Heuler, M Barkhoff, and F. Duisen, Int. J. Fatigue, 104, 7280 (2017)

[10] F. Grasland, J. M. Chenal, L. Chazeau, J. Caillard, and R. Schach, Proc. Tenth Eur. Conf. Const. Models Rubber, 65 (2017)

[11] M. Johlitz and A. Lion, Contin. Mech. Thermodyn., 25, 605-624 (2013)

[12] M. Celina, K. T. Gillen, and R. A. Assink, Polym. Degrad. Stab., 90, 395-404 (2005)

[13] J. T. South, S. W. Case, and K. L. Reifsnider, Rubber Chem. Technol., 76, 785-802 (2003)

[14] P. Charrier, Y. Marco, V. Le Saux, and R. K. P. S. Ranaweera, Proc. Seventh Eur. Conf. Const. Models Rubber, 381 (2011)

[15] J. Verdu, Oxidative ageing of polymers (WileyISTE, 2012)

[16] M. Celina, J. Wise, D. K. Ottesen, K. T. Gillen, and R. L. Clough, Polym. Degrad. Stab., 68, 171$184(2000)$

[17] V. Le Saux, Fatigue et vieillissement des élastomères en environnements marin et thermique : de la caractérisation accélérée au calcul de structure, Université de Bretagne occidentale, France (2010)

[18] G. T. Kohman, J. Phys. Chem., 33, 226-243 (1929)

[19] J. R. Shelton and H. Winn, Ind. Eng. Chem., 36 728-730 (1944)

[20] S. G. Kim and S.-H. Lee, Rubber Chem. Technol., 67, 649-661 (1994)

[21] P. Soma, N. Tada, M. Uchida, K. Nakahara, and Y. Taga, J. Solid Mech. Mater. Eng., 4, 727-737 (2010)

[22] P. D. Calvert and N. C. Billingham, J. Appl. Polym. Sci., 24, 357-370 (1979)

[23] A. Roychoudhury and P. P. De, J. Appl. Polym. Sci., 50, 181-186 (1993)

[24] S. T. Tchalla, P. Y. Le Gac, R. Maurin, and R. Créac'hcadec, Polym. Degrad. Stab., 139, 28-37 (2017)

[25] G. Spetz, Polym. Test., 13, 239-270 (1994)

[26] ASTM D 1765, Standard classification system for carbon blacks used in rubber products (2016)

[27] NF ISO 37, Caoutchouc vulcanisé ou thermoplastique. Détermination des caractéristiques de contrainte-déformation en traction (2012)

[28] E. Ostoja Kuczynski, P. Charrier, E. Verron, G. Marckmann, L. Gornet, and L. Chagnon, Proc. Third Eur. Conf. Const. Models Rubber (2003)

[29] M. Celina, J. Wise, D. K. Ottesen, K. T. Gillen, and R. L. Clough, Polym. Degrad. Stab., 60, 493504 (1998)

[30] K. T. Gillen, R. Bernstein, and D. K. Derzon, Polym. Degrad. Stab., 87, 57-67 (2005)

[31] J. T. South, Mechanical properties and durability of natural rubber compounds and composites, Faculty of the Virginia Polytechnic Institute, United-States (2001) 